\title{
Analysis of Low-Density Lipoprotein Receptor Gene Mutations in A Family with Familial Hypercholesterolemia
}

\section{Ya-nan $\mathrm{Hu}$}

Shengli Clinical Medical College of Fujian Medical University

Min Wu

Shengli Clinical Medical College of Fujian Medical University

Yuan-yuan Ji

Shengli Clinical Medical College of Fujian Medical University

Jian-Hui Zhang

Shengli Clinical Medical College of Fujian Medical University

Yan-ping Zhang

Shengli Clinical Medical College of Fujian Medical University

\section{Qiu-yan Wu}

Shengli Clinical Medical College of Fujian Medical University

\section{Xin-fu Lin}

Fujian provincial hospital

Jia-bin Wu

Fujian provincial hospital

\section{Li-sheng Liao}

Fujian provincial hospital

Jie-Wei Luo ( $\nabla$ docluo0421@aliyun.com )

Fujian provincial hospital

\section{Xin Chen}

Fujian provincial hospital

\section{Research Article}

Keywords: Hypercholesterolemia, LDLR, LDL-C, gene mutation, mutation site

Posted Date: February 17th, 2022

DOI: https://doi.org/10.21203/rs.3.rs-1353487/v1 
License: (c) (i) This work is licensed under a Creative Commons Attribution 4.0 International License. Read Full License 


\section{Abstract}

Background: Familial hypercholesterolemia $(\mathrm{FH})$ is a common monogenic autosomal dominant hereditary disease, mainly caused by pathogenic mutations in the low-density lipoprotein receptor ( $L D L R)$ gene. It is characterized by severely elevated LDL cholesterol (LDL-C) levels, cutaneous xanthomatosis, and premature coronary heart disease.

Materials and Methods: Whole exome sequencing of the proband with familial hypercholesterolemia was performed to identify the target gene and screen for suspicious pathogenic mutations. Sanger sequencing of family members was conducted to analyze family genetic-phenotypic associations. In addition, the changes in the structure and function of mutant LDLR were predicted using biological information analysis.

Results: Next Generation Sequencing revealed that the proband carried two heterozygous missense point mutations c.G1027A (p.G343S) and c.G1879A (p.A627T) in LDLR (NM_000527), which could lead to FH syndrome. In this pedigree, the proband and her brother with hypercholesterolemia and xanthelasma carried the compound heterozygous mutations p.G343S and p.A627T. Her father, children, and nephew carried the p.G343S mutation, and her mother and niece carried the p.A627T mutation. The total cholesterol (TC) of a compound heterozygote was 20.50-21.35 mmol/L, the cholesterol of a heterozygote was $6.52-8.81 \mathrm{mmol} / \mathrm{L}$, and the TC of other family members without a mutation was 2.94$3.61 \mathrm{mmol} / \mathrm{L}$. G343S and A627T had a slight effect on the protein structure of LDLR, as simulated by Chimera. In addition, Rredictprotein software predicted that G343S and A627T affected the disordered region, protein interaction binding region, and RNA binding region of the LDLR. Gly343 and Ala627 are highly conserved in mammals and play a vital role in maintaining the function of the LDLR. YinOYang 1.2 predicted that these two sites had great potential for O-GIcNac modification, and GPS 5.0 predicted that the variants modified phosphorylation. Mutations may affect protein post-translational modification, thus changing the structure, function, location, and fate of the protein.

Conclusions区The mutations c.G1027A in LDLR exon 7 and c.G1879A in exon 13 may be the molecular genetic basis of $\mathrm{FH}$ in this family. Compared to patients with a heterozygous variant, patients with compound heterozygous variants had much higher levels of cholesterol and had xanthoma

\section{Background}

Familial hypercholesterolemia $(\mathrm{FH})$ is a common monogenic autosomal hereditary disease that is dominantly inherited, except for autosomal recessive hypercholesterolemia (ARH), an extremely rare disease ${ }^{1}$. FH is most frequently caused by variations in the low-density lipoprotein receptor $(L D L R)$ gene 2,3 , which is characterized by severely elevated blood total cholesterol(TC) levels, especially LDL cholesterol (LDL-C), multiple cutaneous xanthomatosis, valvular regurgitation, arcus cornea, and premature coronary heart disease ${ }^{4,5}$. Owing to long-term exposure to high levels of LDL-C from birth, patients with FH may have an increased risk of atherosclerotic cardiovascular disease (ASCVD) and even 
myocardial infarction ${ }^{6}$. The risk of premature coronary heart disease in $\mathrm{FH}$ is 20 -fold higher than that in average people ${ }^{7}$. Patients with FH develop ASCVD earlier and have more severe and diverse clinical manifestations than those with other types of hyperlipidemia. Full application of genetic diagnostic techniques and improvement of $\mathrm{FH}$ disease databases are of great significance for the screening and prevention of early $\mathrm{FH}$.

Most of the autosomal dominant variations are found in $L D L R$, proprotein convertase subtilisin/kexin type 9 (PCSK9), and apolipoprotein $\mathrm{B}(A P O B)^{8,9}$. Low-density lipoprotein receptor adaptor protein 1 (LDLRAP1) has been linked to $A R H$, which is involved in the endocytosis of LDL receptors ${ }^{10}$. Human $L D L R$ was first cloned in 1983. It is located on the distal short arm of chromosome 19 at 19p13.1-13.3, spans 45 kilobases, and has 18 exons and 17 introns ${ }^{11}$. $A P O B$ is the major apolipoprotein on lipoprotein molecules and serves as a ligand for the LDLR ${ }^{12}$. PCSK9 encodes a serine protease, which regulates the degradation of the LDLR in lysosomes and prevents its recycling to the cell surface. PCSK9 is mainly secreted by the liver, binds to the LDLR on the hepatocyte surface to form a tight complex, and enters the lysosome for degradation, which reduces LDLR levels on the hepatocyte surface and reduces the

clearance of LDL-C by the liver ${ }^{12,13}$. Goldstein and Brown ${ }^{14}$ first reported in 1951 that FH pathogenesis is chiefly due to the structural loss and functional abnormality of the LDLR on the cell membrane surface caused by mutations in the gene encoding the LDLR. These mutations affect LDLR binding to LDL containing $A P O B$ and $A P O E$, resulting in the disturbance of LDL metastasis in blood and excessive siltation in tissues, leading to multiple cutaneous xanthoma and atherosclerosis. The prevalence of heterozygous $\mathrm{FH}(\mathrm{HeFH})$ is $1: 200-300$, whereas homozygous $\mathrm{FH}(\mathrm{HoFH})$ presents in a proportion of 1:170,000-300,000 individuals ${ }^{15}$. Generally, serum TC levels in heterozygous patients are between 6.5 and $14.3 \mathrm{mmol} / \mathrm{L}$, and LDL-C levels are $>4.9 \mathrm{mmol} / \mathrm{L}^{16}$, while serum TC levels in homozygous patients are usually $>18 \mathrm{mmol} / \mathrm{L}$ and LDL-C levels are $>13 \mathrm{mmol} / \mathrm{L}^{17}$.

In this study, a FH family with coronary heart disease and cutaneous xanthomatosis caused by compound heterozygous mutations in $L D L R$ was identified using whole exome gene capture sequencing technology. Phenotypic investigation and phenotype-gene mutation association analysis of the family was performed, and the structure and function of the mutant protein was predicted using bioinformatics tools to provide a reference for researchers.

\section{Results}

\section{Pedigree clinical data}

There were 14 members in the family, including five males and nine females, and eight had hypercholesterolemia, including the proband. The proband had recurrent chest tension for 20 years, and the patient had a soybean-sized xanthoma on the left upper eyelid with a corneal arcus (Fig. 1b). After admission, routine blood tests, liver function, renal function, electrolytes, and blood glucose tests were normal. Autoantibodies and anti-O antibodies were negative. An immune panel, erythrocyte 
sedimentation rate, and C-reactive protein levels were normal. Thyroid function (thyroid stimulating hormone, triiodothyronine, and thyroxine) was unremarkable, and an electrocardiogram showed no obvious abnormalities. Blood lipid tests revealed the following levels: serum TC $20.50 \mathrm{mmol} / \mathrm{L}(<5.18$ $\mathrm{mmol} / \mathrm{L}$ ), triglyceride (TG) $1.45 \mathrm{mmol} / \mathrm{L}$ ( $<1.7 \mathrm{mmol} / \mathrm{L}$ ), LDL-C $18.80 \mathrm{mmol} / \mathrm{L}$, high-density lipoprotein cholesterol (HDL-C) $1.26 \mathrm{mmol} / \mathrm{L}(1.29-1.55 \mathrm{mmol} / \mathrm{L})$, apolipoprotein A (apoA) $1.53 \mathrm{~g} / \mathrm{L}(1.2-1.6 \mathrm{~g} / \mathrm{L})$, apolipoprotein $\mathrm{B}(\mathrm{apoB}) 0.97 \mathrm{~g} / \mathrm{L}(0.6-1.1 \mathrm{~g} / \mathrm{L})$. Coronary computed tomography angiography (CTA) revealed segmental eccentric mixed plaques in the wall of the proximal segment of the left main coronary artery to the anterior descending branch, with stenosis of approximately $70 \%-80 \%$. Echocardiography revealed left ventricular enlargement, moderate mitral insufficiency, and decreased coronary flow reserve. Carotid artery color Doppler ultrasound revealed plaques at the origin of the bilateral common carotid artery and diffuse thickening of the intima of the middle artery. According to the British Simon criteria ${ }^{18}$ and the Dutch Network Diagnostic Criteria ${ }^{19}$ for FH diagnosis, combined with family history and subsequent gene sequencing, the proband was diagnosed with $\mathrm{FH}$, with a compound heterozygous mutation and coronary atherosclerotic heart disease.

The younger brother of the proband presented with cutaneous xanthoma and coronary atherosclerotic heart disease (Fig. 1c). Eight individuals, including the proband (II2), had hypercholesterolemia (I1, I2, II2, $I I 7, I I 11, I I I 2, I I I 7$, and III8). Carotid color ultrasound of the proband's father (I1) revealed plaques at the bifurcation of the left common carotid artery. Doppler ultrasound of the carotid artery of the proband's younger brother (II7) revealed bilateral common carotid artery plaques and right subclavian artery plaques, and echocardiography revealed large, mild mitral and tricuspid valves. Coronary CTA showed segmental eccentric mixed plaques in the wall of the proximal segment from the left main trunk to the anterior descending branch, with a stenosis of approximately $65 \%-75 \%$. No obvious abnormalities were observed among other family members. The plasma cholesterol of four patients met the FH diagnostic criteria, in which the TC and LDL-C values of the proband and his younger brother were equivalent to those of homozygous $\mathrm{FH}(\mathrm{HoFH})$, while the father and son had heterozygous $\mathrm{FH}(\mathrm{HeFH})$. Serum total cholesterol was $20.50-21.35 \mathrm{mmol} / \mathrm{L}$ for family members carrying compound heterozygote mutations (III, III), 6.52-8.81 mmol/L for family members carrying one heterozygote mutation (I1, I2, III1, III2, III7, III8), and 2.94-3.61 mmol/L for family members with no mutations (II3-6, II8, III3-6) (Table 1).

\section{High-throughput genome sequencing and verification using Sanger sequencing}

Target enrichment with next-generation sequencing was used to scan pathogenic gene variants and abnormal gene copy numbers (copy number variation analysis) in all exons of the genome. To identify pathogenic variants in the proband's genome, single nucleotide polymorphisms (SNPs) with a minor allele frequency $>1 \%$ were filtered out. A heterozygous missense variant of c.G1027A was identified in exon 7 of $L D L R$ (NM_000527) and a heterozygous missense variant of c.G1879A was also identified in exon 13; these mutations were verified using Sanger sequencing (Fig. 1d-g). The c.G1027A and c.G1879A variants transform glycine (Gly) to serine (Ser) and alanine (Ala) to threonine ( $\mathrm{Thr}$ ) at residues 343 and 627, respectively, of the LDLR. According to the American College of Medical Genetics and Genomics (ACMG) pathogenicity rating criteria and guidelines, it was predicted that the c. G1027A mutation was 
pathogenic and the c.G1879A mutation was likely to be pathogenic; thus, these mutations were added to the ClinVar database. Sanger sequencing of family members showed that the proband and her brother (II7) carried compound heterozygous missense mutations: c.1027G > A (p.Gly343Ser) and c.1879G > A (p.Ala627Thr), whereas I1, III1, III2, and III8 only carried the c. G1027A mutation, and I2 and III7 carried the c. G1879A mutation. No mutations were identified in other family members (Fig. 1a).

\section{Structure and function prediction of the LDLR}

The interaction diagram between the LDLR and other proteins was plotted using String and the X-ray crystal diffraction structure of the LDLR with the interaction protein proprotein convertase subtilisin/kexin type 9 (PCSK9) was plotted using Swiss-Model (Fig. 2a and 2b). The position of the mutation site on the three-dimensional structure of the LDLR is shown in Fig. 2b. The protein structure near the two mutation sites was simulated using Chimera, which showed that these two mutations had a slight effect on LDLR protein structure (Fig. $2 \mathrm{c}$ and 2 d). In addition, the effect of the two mutation sites on the functional domain of the protein was predicted using Rredictprotein software. G343S and A627T affected the disordered region, protein interaction binding region, and RNA binding region of the LDLR, suggesting that the two point mutations might affect the properties and function of the protein (Fig. 3).

Protein sequence conservation analysis showed that the mutation sites Gly343 and Ala627 were highly conserved in mammals, and the amino acid mutation near Gly343 would cause severe LDLR-deficient disease, indicating that these two amino acid sites played an important role in the maintenance of LDLR function (Fig. 4a). Phosphorylation modification of these two sites was predicted using GPS 5.0. G343S was recognized and phosphorylated by protein kinase signaling pathways, such as IKB kinase and cAMPdependent protein kinase A/cGMP-dependent protein kinase G/protein kinase C (Fig. 4b), while A627T was recognized and phosphorylated by protein kinase signaling pathways, such as sterile and tyrosine kinase-like (Fig. 4c). In addition, A627T might affect phosphorylation at S630 (Fig. 4d). YinOYang 1.2 was used to predict that these two sites might undergo O-GIcNac modification (Fig. 4e). The potential changes in protein post-translational modification of these two mutation sites and nearby amino acids might change the structure, function, localization, and fate of the proteins, leading to functional defects in the LDLR.

\section{Discussion}

$\mathrm{FH}$ is the most common congenital lipid metabolism disorder, which is primarily due to gene mutations related to the LDL uptake pathway mediated by the LDLR, resulting in abnormal LDLR structure or function on the cell membrane surface and the inability to effectively metabolize LDL in the body. This results in hypercholesterolemia caused by excessive LDL stasis in the blood or other tissues due to impaired clearance ${ }^{20,21}$. If left untreated, this cholesterol metabolism genetic disorder may lead to premature atherosclerosis, coronary artery disease (CAD), and even death at a very young age ${ }^{16}$. The average age of patients with $\mathrm{HeFH}$ and CAD is $<45$ years, in men and $<55$ years in women ${ }^{22,23}$, while in patients with $\mathrm{HoFH}$, coronary heart disease may present at the age of 20 years ${ }^{24}$. The major cause of 
death in $\mathrm{FH}$ is coronary heart disease, with a frequency as high as $60 \%$. However, the prevalence of cerebrovascular disease in $\mathrm{HeFH}$ is only $5 \%-10 \% 25$.

Any apparently deleterious type of $L D L R$ variant may impair LDLR function; however, only very particular variants in $A P O B$ and $P C S K 9$ result in $\mathrm{FH}^{8}$. APOB pathogenic variants must allow the synthesis of apolipoprotein, but they need to specifically change the binding affinity to the LDLR to cause disease, such as the known LDL-binding domain located in exons 26 and 29 of APOB ${ }^{8}$. PCSK9 pathogenic variants must enhance the function of the encoded protein and promote the degradation of the LDLR, which causes $\mathrm{FH}^{26,27}$; $\mathrm{FH}$ is most frequently caused by variations in $L D L R$. ClinVar contains more than 4,970 mutants in $L D L R, 580$ mutants in $A P O B$, and 350 mutants in $P C S K 9^{28}$. $L D L R$ mutations are detected in $80 \%-85 \%$ of $\mathrm{FH}$ cases, $A P O B$ mutations are identified in $5 \%-7 \%$ of patients, and PCSK 9 gene mutations are detected in less than $5 \%$ of cases, while LDLRAP1 mutations are detected in less than $1 \%$ of cases ${ }^{23}$. The severity of hypercholesterolemia and the risk of CAD in patients with $\mathrm{FH}$ are associated with the type of gene mutation, where $L D L R$ nonsense mutations are the most severe, while $A P O B$ and $P C S K 9$ causative mutations usually have a mild phenotype ${ }^{26}$. LDL-C levels were significantly higher in patients with $\angle D L R$ pathogenic mutations than those in patients with $A P O B$ and $P C S K 9$ pathogenic mutations $9,16,29$. The degree of atherosclerosis depends more on the plasma LDL-C level than on the affected gene or mutation type ${ }^{16}$.

Missense variants are the most prevalent type of variant in $\angle D L R, A P O B$, and $P C S K 9$, followed by frameshift mutations in $\angle D L R$, and synonymous mutations in $A P O B$ and $P C S K 9^{8}$. In $\angle D L R, 18 \%$ of the variants are located in exon 4. In $A P O B, 41 \%$ of variants are located in exon 26 , and $15 \%$ are located in exon 29, while in PCSK9, $19 \%$ of variants are located in the 3 ' non-coding region ${ }^{8}$. As of October 2021 , the FH disease database contains more than 3,800 LDLR-causing mutations (www.ucl.ac.uk/fh). Large-scale DNA copy number variations account for approximately $8 \%-10 \%$ of the reported $L D L R$ mutations, whereas nonsense mutations in the coding regions account for $12 \%-15 \%$ of cases ${ }^{16}$. Small insertions or deletions in or near coding sequences that may or may not alter the reading frame account for $15 \%-20 \%$ of mutations, whereas splicing mutations, usually non-coding, appear at intron-exon boundaries in $8 \%-$ $10 \%$ of mutations ${ }^{16}$. LDLR mutations are predominantly located in exon 4 , which may be because exon 4 is the largest exon in $L D L R$ or because variants in this exon encoding the ligand-binding domain have a highly deleterious effect on gene function ${ }^{12}$. On the contrary, the mutation frequencies of exons 15 and 16 are extremely low ${ }^{12}$. However, the impact of these mutations on $\mathrm{FH}$ pathophysiology has not been fully elucidated.

The distribution characteristics of $L D L R$ mutations vary in different regions and ethnic groups, which may be related to founder effects ${ }^{11,30,31}$. In Japanese and East Asian populations, the frequency of the $L D L R$ c. $344 \mathrm{G}>\mathrm{A}$ (p.Arg115His) mutation is relatively high compared to that of other causative variants ${ }^{28}$. In central Japan, $L D L R$ variants are frequently found in c. 2431A > T, c.2312-3C > A, and deletion of exons 2-3 ${ }^{9}$. In Mexico, $34 L D L R$ causative variants have been reported, of which, p.Leu759Serfs 6 is the most 
common mutation ${ }^{32}$. In the Greek population, $41 \angle D L R$ gene variants have been identified, among which $L D L R$ c. $81 \mathrm{C}>\mathrm{G}, \mathrm{c} .517 \mathrm{~T}>\mathrm{C}, \mathrm{c} .858 \mathrm{C}>\mathrm{A}, \mathrm{c} .1285 \mathrm{G}>\mathrm{A}, \mathrm{c} .1646 \mathrm{G}>\mathrm{A}$ and $\mathrm{c} .1775 \mathrm{G}>\mathrm{A}$ variants accounts for more than $80 \%$ of all variants ${ }^{33}$. In Chinese individuals, most of the variants in $L D L R$ are located in exon 4 , and nearly $60 \%$ are missense mutations ${ }^{34}$, of which c. $986 \mathrm{G}>\mathrm{A}, \mathrm{c} .1747 \mathrm{C}>\mathrm{T}, \mathrm{c} .1879 \mathrm{G}>\mathrm{A}$, and c. $268 \mathrm{G}$ $>A$ are the most common mutation types, and the frequency of the four mutations is $>4 \%{ }^{35}$. In addition, p.Asp206Glu, p.Val408Met, and p.Asp154Asn are highly prevalent in white South Africans ${ }^{30,32}$.

The LDLR is a type 1 transmembrane protein composed of 860 amino acids, including a 21-residue signal peptide ${ }^{36}$. The LDLR has five functional domains, and the ectodomain is composed of three domains: an N-terminal ligand-binding domain, a domain highly homologous to epidermal growth factor precursor, and a domain enriched in O-linked sugar. The transmembrane domain consists of 22 residues, and the cytoplasmic region consists of a cytoplasmic domain of 50 residues ${ }^{36}$. Exon 1 of $L D L R$ encodes a signal sequence, exons 2-6 encode the ligand-binding domain consisting of seven cysteine-rich tandem repeats of a 40-amino acid residue, and exons 7-14 encode domains that are highly homologous to the human epidermal growth factor precursor (pro-EGF). This domain contains three cysteine-rich growth factor repeats, consisting of 40 residues ${ }^{11}$. Exon 15 encodes a 58-amino acid sequence, which is the attachment site for many 0-linked carbohydrate chains, whereas exon 16 and the 5 -end of exon 17 encode 22 hydrophobic amino acids that anchor the protein to the cell membrane ${ }^{11}$. The rest of exon 17 and the 5 -end of exon 18 encode a cytoplasmic domain that contains the motifs required for concentrating the LDLR in clathrin-coated pits, enabling their internalization by receptormediated endocytosis ${ }^{11}$. When the 21 -residue signal peptide is cleaved, the mature LDLR is inserted into the endoplasmic reticulum (ER) membrane, where the ectodomain is folded and glycosylated ${ }^{37}$. Properly folded LDLR exits the ER, N-linked sugars are modified, and O-linked sugars are added to the LDLR in the Golgi, resulting in an increase in the apparent molecular weight from 120 to $160 \mathrm{kDa}{ }^{36,37}$. After transport to the cell membrane, the LDLR is concentrated in caged protein-encapsulated pits ${ }^{37}$.

$L D L R$ was principally transcriptionally expressed and translated into functional proteins in tissues, such as the testis, colon, kidney, liver, lung, and stomach (Fig. 5a and 5b, data from The Human Protein Atlas database), which was consistent with the metabolic site of LDL. CRISPR screening technology data was analyzed based on genome-wide data (https://orcs.thebiogrid.org/Gene/3949) to validate the potential physiological functions of the LDLR. LDLR deletion had an important impact on the metabolism of cells and individuals, especially cholesterol metabolism and viral immune responses, indicating that LDLR played a complex, but essential biological role (Fig. 5c). The Alliance of Genome Resources (https://www.alliancegenome.org/gene/HGNC:6547) indicates that loss of LDLR function not only causes cardiovascular diseases, such as $\mathrm{FH}$, xanthomatosis, and atherosclerosis, but also leads to diseases, such as Alzheimer's disease, migraine without aura, and systemic lupus erythematosus, also indicating that LDLR is necessary to maintain normal physiological metabolic function in the body (Supplyment 1). Furthermore, it was observed that LDLR was poorly expressed in many cancers, such as liver cancer (Fig. 6a and 6b, data from The Cancer Genome Atlas, Gene Expression Profiling Interactive 
Analysis 2, and The Human Protein Atlas databases). Single-cell transcriptome sequencing data of hepatocellular carcinoma tissues revealed that the LDLR was mainly expressed in biliary epithelial cells and hepatocytes (Fig. $6 \mathrm{c}$ and $6 \mathrm{~d}$ ), suggesting that the LDLR may play an important role in hepatocarcinogenesis.

Normally, LDL in the extracellular matrix is recognized by the LDLR on the cell membrane surface, transported into the cell, and eventually degraded by lysosomes. LDL entering the cell also inhibits the activity of 3-hydroxy-3-methylglutaryl-CoEnzyme A in microsomes, thereby inhibiting cholesterol synthesis in vivo. Abnormal LDLR causes increased LDL accumulation in the extracellular matrix and plasma, and negative feedback leads to increased efficiency of LDL synthesis, further increasing extracellular LDL levels. According to the location and nature of the mutations, LDLR function-defective mutations are chiefly divided into five types ${ }^{38}: 1$ ) Null alleles that are unable to transcribe $L D L R \mathrm{mRNA}$ or translate LDLR protein, including representative mutations, such as promoter mutations, translation initiation codon mutations, translation termination codon mutations, or premature translation termination and frameshift mutations; 2) Transport-deficient alleles, where successfully translated LDLR cannot be localized to the cell membrane, resulting in its accumulation in the cell or secretion outside the cell, including representative mutations, such as signal peptide mutations and transmembrane domain mutations; 3) Binding defective alleles, where the LDLR located on the cell membrane cannot bind LDL in the extracellular matrix; 4) Transport defective alleles, where LDLR binding to LDL cannot transport LDL from the extracellular matrix to the cell interior; and 5) Recycling defective alleles, where the LDLR carrying LDL into the cell is unable to return to the cell membrane surface through membrane trafficking. Another pathogenic mode, as a sixth class of mutations in the LDLR, has been reported where mutations in the LDLR transmembrane domain interfere with the normal insertion of the LDLR in the cell membrane. The mutant LDLR protein is cleaved by metalloproteases in the ER, causing shedding of the ectodomain or rapid degradation at the cell surface, leading to a reduced number of LDLRs on the membrane ${ }^{36,37}$.

The severity of hypercholesterolemia in patients with $\mathrm{FH}$ is related both to the function of the mutant protein, and to the number of defective alleles ${ }^{39}$. Pathogenic mutations in $L D L R$ are codominant and have a genetic dosage effect ${ }^{21}$. In addition, in a cross-sectional analysis in the Netherlands, the prevalence of type 2 diabetes $(1.73 \%)$ in patients with $\mathrm{FH}$ was evidently lower than that in unaffected relatives $(2.93 \%)$ and varied by mutation type. This study investigated the possibility of a causal relationship between LDLR-mediated transmembrane cholesterol transport and type 2 diabetes ${ }^{40}$.

Despite the high incidence of $\mathrm{FH}, \mathrm{FH}$ has been severely neglected and undertreated. It is estimated that > $90 \%$ of the 30 million patients with $\mathrm{FH}$ worldwide are undiagnosed ${ }^{26}$. Therefore, it is crucial to popularize $\mathrm{FH}$ health knowledge and improve the $\mathrm{FH}$ diagnosis rate. Cascading genetic screening is the most effective method to identify patients with $\mathrm{FH}$ and is a cost-effective means to prevent coronary heart disease, myocardial infarction, and death in patents with $\mathrm{FH}^{41-44}$. Cascade genetic screening might identify at-risk relatives of patients diagnosed with $\mathrm{FH}$ through interfamilial genetic patterns, help early 
and accurate detection of patients with $\mathrm{FH}$, and provide timely treatment, thereby improving the prognosis of patients and achieving primary prevention of cardiovascular disease.

Several limitations merit discussion. First, the research requires in vitro molecular analysis to examine the impact on transcription or splicing and further assessing the efficacy of medication treatment for $\mathrm{FH}$ patients. Moreover, the sample size was limited.

\section{Conclusion}

In conclusion, the mutations c.G1027A and c.G1879A in LDLR were preliminarily confirmed to be the molecular genetic basis of $\mathrm{FH}$ in this family. We predicted the effect of the mutations on the protein structure, modification and function of the LDLR, providing a basis for these two mutations affecting the normal LDLR function. This study illustrated the clinical significance of LDLR gene mutations, and analyzed the relationship between the pathogenic variants and the clinical phenotype of $\mathrm{FH}$, enriched the spectrum of $\mathrm{FH}$, and provided more accurate and effective data support for the diagnosis and treatment of $\mathrm{FH}$ in the future.

\section{Methods}

\section{Research subjects:}

Proband: male, 53-year-old Chinese Han, in Fujian, China. The chief complaint was "recurrent chest tension for 20 year" and the patient was found to have a soybean-sized xanthoma on the left upper eyelid with a corneal arcus. Multiple examinations of plasma lipids showed a remarkable increase, and coronary CTA revealed segmental eccentric mixed plaques in the wall of the proximal segment of the left main artery to the anterior descending branch, with stenosis of approximately $70 \%-80 \%$. A similar medical history was found in the family, with no consanguineous marriage in the family. Routine physical examination, blood biochemistry, electrocardiogram, carotid ultrasound, echocardiography, and coronary CTA were performed on the other 13 family members. Blood and biochemical test data were analyzed and a genetic map of the family was recorded. This study was approved by the Ethics Committee of Fujian Provincial Hospital, and all family members who participated in this study provided signed informed consent.

\section{Candidate gene mapping and variant screening strategy}

Peripheral blood was collected from the proband in an EDTA anticoagulant tube, and DNA was extracted according to the instructions of the QIAamp DNA Blood Mini Kit (QIAGEN, Cat No. 51106; Qiagen, Hilden Germany). The quality of the extracted DNA was determined using a NanoDrop instrument (Thermo Fisher, Waltham, MA, USA). The TargetSeq ${ }^{\circledR}$ liquid phase probe hybridization capture technique developed by iGeneTech ${ }^{\circledR}$ (Beijing, China) was used to build a genomic DNA library capturing the exon regions of 20,000 genes associated with genetic diseases. The PE150 sequencing strategy was used on a NovaSeq 6000 platform capable of performing parallel gene analyses. The target genes in this panel 
included PCSK9, APOB, LDLR, LDLRAP1, ATP-binding cassette sub-family G member 5 (ABCG5), and $A B C G 8$. After alignment against the reference Human hg19 genome sequence, GATK and ANNOVAR were used to find and analyze single-nucleotide variants and small indels in the sequencing data. Using human genome information from databases, such as dbSNP, 1000 Genomes (1000G), the Human Gene Mutation Database, and Exome Sequencing Project v. 6500 (ESP6500), variants were screened and annotated according to the ACMG guidelines. The filtered and separated sequenced reads were aligned with the hg19 sequence using the Burrows-Wheeler Aligner, and variant information was collected on gene features, variant type, 1000G, and ESP6500 frequency. Polymorphism Phenotyping (PolyPhen-2, http://genetics.Bwh.harvard.edu/ppH2/), sorting intolerant from tolerant (SIFT, http://sift.jcvi.org/), Protein Variation Effect Analyzer (PROVEAN, http://provean.jcvi.org/protein_batch_submit.php? species=human), and mutation taster (http://mutationtaster.org/) were used to predict the pathogenicity of these variants and their biological significance. Primers for amplification of the suspect variant sites were designed using Primer Premier 5.0. Sanger sequencing was performed on an ABI 3500 Dx platform to verify the NovaSeq 6000 sequencing results. The amplified fragment of the target sequence of the LDLR (NM_000527) c.G1027A variant was 322 bp. The primers used were F:

GAGTGACCAGTCTGCATCCC and R: AAACTCTGGCCAGCCAATGA, with an annealing temperature of 60 ${ }^{\circ} \mathrm{C}$. The amplified fragment length of the target sequence of the $L D L R$ c.G1879A variant was $1122 \mathrm{bp}$. The primers used were F: GAAATGGATGGTGGTGATG and R囚GGGCAGAAGAAGCGGAGT, and the annealing temperature was $60^{\circ} \mathrm{C}$. Primers were synthesized by Genokon Medical (Xiamen, China).

\section{Abbreviations}

FH:Familial hypercholesterolemia;LDLR:low-density lipoprotein receptor;LDL-C:LDL cholesterol;TC:total cholesterol;ARH:autosomal recessive hypercholesterolemia;ASCVD:atherosclerotic cardiovascular disease; PCSK9: proprotein convertase subtilisin/kexin type 9;APOB:apolipoprotein B; LDLRAP1:Low-density lipoprotein receptor adaptor protein 1; HeFH:heterozygous FH; HoFH:homozygous FH; TG:triglyceride; HDL-C: high-density lipoprotein cholesterol; apoA:apolipoprotein A; CTA: computed tomography angiography;SNPs:single nucleotide polymorphisms;

Gly:glycine;Ser:serine;Ala:alanine;Thr:threonine;ACMG:American College of Medical Genetics and Genomics; CAD:coronary artery disease; ER:endoplasmic reticulum; ABCG:ATP-binding cassette subfamily $\mathrm{G}$ member.

\section{Declarations}

\section{Ethics approval and consent to participate}

This study was approved by the Ethics Committee of Fujian Provincial Hospital (K2015-031-01). All family members participating in this study provided informed consent.

\section{Consent for publication}


Not applicable.

\section{Availability of data and materials}

Data supporting the findings of this study are available from the corresponding author upon reasonable request.

\section{Competing interests}

The authors declare that they have no competing interests

\section{Funding}

This work was supported by Fujian Province Medical Innovation Foundation (2019-CXB-4), Natural Science Foundation of Fujian Province (2021 J02053) and the Special Research Foundation of Fujian Provincial Department of Finance(2020-500\#), P.R. China.

\section{Authors' contributions}

Jie-Wei Luo and Xin Chen designed the study. Min Wu, Yuan-yuan Ji, Jian-Hui Zhang, Yan-ping Zhang performed the data collection. Qiu-yan Wu, Xin-fu Lin, Li-sheng Liao and Jia-bin Wu performed the data analysis and interpretation of results. Ya-nan Hu wrote the original draft. Jie-Wei Luo reviewed and provided critical comments on the manuscript. All authors read and approved the final version of the manuscript.

\section{Acknowledgements}

Not applicable.

\section{References}

1 Harada-Shiba, M. et al. Guidelines for Diagnosis and Treatment of Familial Hypercholesterolemia 2017. J Atheroscler Thromb25, 751-770, doi:10.5551/jat.CR003 (2018).

2 Leigh, S. et al. The UCL low-density lipoprotein receptor gene variant database: pathogenicity update. $J$ Med Genet54, 217-223, doi:10.1136/jmedgenet-2016-104054 (2017).

3 Saint-Jore, B. et al. Autosomal dominant type lla hypercholesterolemia: evaluation of the respective contributions of LDLR and APOB gene defects as well as a third major group of defects. Eur $J$ Hum Genet8, 621-630, doi:10.1038/sj.ejhg.5200516 (2000).

4 Ghosh, S., Majumder, B. \& Dutta, A. Tuberous xanthoma as a presenting feature of familial homozygous hypercholesterolemia with aortic regurgitation. The Journal of pediatrics166, 198, doi:10.1016/j.jpeds.2014.09.022 (2015). 
5 Mabuchi, H. et al. Molecular genetic epidemiology of homozygous familial hypercholesterolemia in the Hokuriku district of Japan. Atherosclerosis214, 404-407, doi:10.1016/j.atherosclerosis.2010.11.005 (2011).

6 Singh, S. \& Bittner, V. Familial hypercholesterolemia-epidemiology, diagnosis, and screening. Curr Atheroscler Rep17, 482, doi:10.1007/s11883-014-0482-5 (2015).

7 Watts, G. F., Lewis, B. \& Sullivan, D. R. Familial hypercholesterolemia: a missed opportunity in preventive medicine. Nat Clin Pract Cardiovasc Med4, 404-405, doi:10.1038/ncpcardio0941 (2007).

8 lacocca, M. A. et al. ClinVar database of global familial hypercholesterolemia-associated DNA variants. Hum Mutat39, 1631-1640, doi:10.1002/humu.23634 (2018).

9 Hori, M. et al. Impact of LDLR and PCSK9 pathogenic variants in Japanese heterozygous familial hypercholesterolemia patients. Atherosclerosis289, 101-108, doi:10.1016/j.atherosclerosis.2019.08.004 (2019).

10 D'Erasmo, L., Di Costanzo, A. \& Arca, M. Autosomal recessive hypercholesterolemia: update for 2020. Current opinion in lipidology31, 56-61, doi:10.1097/mol.0000000000000664 (2020).

11 Hobbs, H. H., Brown, M. S. \& Goldstein, J. L. Molecular genetics of the LDL receptor gene in familial hypercholesterolemia. Hum Mutat1, 445-466, doi:10.1002/humu.1380010602 (1992).

12 Sharifi, M., Futema, M., Nair, D. \& Humphries, S. E. Genetic Architecture of Familial Hypercholesterolaemia. Curr Cardiol Rep19, 44, doi:10.1007/s11886-017-0848-8 (2017).

13 Lagace, T. A. et al. Secreted PCSK9 decreases the number of LDL receptors in hepatocytes and in livers of parabiotic mice. J Clin Invest116, 2995-3005, doi:10.1172/JCI29383 (2006).

14 Brown, M. S. \& Goldstein, J. L. Familial hypercholesterolemia: defective binding of lipoproteins to cultured fibroblasts associated with impaired regulation of 3-hydroxy-3-methylglutaryl coenzyme A reductase activity. Proc Natl Acad Sci U S A71, 788-792, doi:10.1073/pnas.71.3.788 (1974).

15 Nohara, A. et al. Homozygous Familial Hypercholesterolemia. J Atheroscler Thromb28, 665-678, doi:10.5551/jat.RV17050 (2021).

16 Defesche, J. C. et al. Familial hypercholesterolaemia. Nat Rev Dis Primers3, 17093, doi:10.1038/nrdp.2017.93 (2017).

17 Banerjee, P. et al. Functional Analysis of LDLR (Low-Density Lipoprotein Receptor) Variants in Patient Lymphocytes to Assess the Effect of Evinacumab in Homozygous Familial Hypercholesterolemia Patients With a Spectrum of LDLR Activity. Arterioscler Thromb Vasc Bio/39, 2248-2260, doi:10.1161/ATVBAHA.119.313051 (2019). 
18 Risk of fatal coronary heart disease in familial hypercholesterolaemia. Scientific Steering Committee on behalf of the Simon Broome Register Group. BMJ303, 893-896, doi:10.1136/bmj.303.6807.893 (1991).

19 Familial hypercholesterolaemia (FH): report of a second WHO consultation, Geneva, 4 September 1998. World Health Organization (1999).

20 Goldberg, A. C. et al. Familial hypercholesterolemia: screening, diagnosis and management of pediatric and adult patients: clinical guidance from the National Lipid Association Expert Panel on Familial Hypercholesterolemia. J Clin Lipido/5, 133-140, doi:10.1016/j.jacl.2011.03.001 (2011).

21 Soutar, A. \& Naoumova, R. Mechanisms of disease: genetic causes of familial hypercholesterolemia. Nature clinical practice. Cardiovascular medicine4, 214-225, doi:10.1038/ncpcardio0836 (2007).

22 Marks, D., Thorogood, M., Neil, H. A. \& Humphries, S. E. A review on the diagnosis, natural history, and treatment of familial hypercholesterolaemia. Atherosclerosis168, 1-14, doi:10.1016/s00219150(02)00330-1 (2003).

23 Mikhailova, S., Ivanoshchuk, D., Timoshchenko, O. \& Shakhtshneider, E. Genes Potentially Associated with Familial Hypercholesterolemia. Biomolecules9, doi:10.3390/biom9120807 (2019).

24 Rodenburg, J. et al. Familial hypercholesterolemia in children. Curr Opin Lipido/15, 405-411, doi:10.1097/01.mol.0000137228.92396.f3 (2004).

$25 \mathrm{Neil}, \mathrm{H}$. A. et al. Non-coronary heart disease mortality and risk of fatal cancer in patients with treated heterozygous familial hypercholesterolaemia: a prospective registry study. Atherosclerosis179, 293-297, doi:10.1016/j.atherosclerosis.2004.10.011 (2005).

26 Sturm, A. C. et al. Clinical Genetic Testing for Familial Hypercholesterolemia: JACC Scientific Expert Panel. J Am Coll Cardio/72, 662-680, doi:10.1016/j.jacc.2018.05.044 (2018).

27 Horton, J. D., Cohen, J. C. \& Hobbs, H. H. Molecular biology of PCSK9: its role in LDL metabolism. Trends Biochem Sci32, 71-77, doi:10.1016/j.tibs.2006.12.008 (2007).

28 Hori, M., Takahashi, A., Son, C., Ogura, M. \& Harada-Shiba, M. The benign c.344G > A: p.(Arg115His) variant in the LDLR gene interpreted from a pedigree-based genetic analysis of familial hypercholesterolemia. Lipids Health Dis19, 62, doi:10.1186/s12944-020-01252-4 (2020).

29 Mabuchi, H. et al. Genotypic and phenotypic features in homozygous familial hypercholesterolemia caused by proprotein convertase subtilisin/kexin type 9 (PCSK9) gain-of-function mutation. Atherosclerosis236, 54-61, doi:10.1016/j.atherosclerosis.2014.06.005 (2014).

30 Steyn, K. et al. Estimation of the prevalence of familial hypercholesterolaemia in a rural Afrikaner community by direct screening for three Afrikaner founder low density lipoprotein receptor gene 
mutations. Hum Genet98, 479-484, doi:10.1007/s004390050243 (1996).

31 Austin, M. A., Hutter, C. M., Zimmern, R. L. \& Humphries, S. E. Genetic causes of monogenic heterozygous familial hypercholesterolemia: a HuGE prevalence review. Am J Epidemio/160, 407-420, doi:10.1093/aje/kwh236 (2004).

32 Hernandez Flores, T. J. et al. LDLR Gene Mutation p.Asp360His and Familial Hypercholesterolemia in a Mexican Community. Arch Med Res51, 153-159, doi:10.1016/j.arcmed.2019.12.017 (2020).

33 Mollaki, V. \& Drogari, E. Genetic causes of monogenic familial hypercholesterolemia in the Greek population: Lessons, mistakes, and the way forward. J Clin Lipido/10, 748-756, doi:10.1016/j.jacl.2016.02.020 (2016).

34 Jiang, L. et al. The distribution and characteristics of LDL receptor mutations in China: A systematic review. Sci Rep5, 17272, doi:10.1038/srep17272 (2015).

35 Tomlinson, B., Hu, M. \& Chow, E. Current status of familial hypercholesterolemia in Chinese populations. Curr Opin Lipido/30, 94-100, doi:10.1097/MOL.0000000000000580 (2019).

36 Strom, T. B., Laerdahl, J. K. \& Leren, T. P. Mutation p.L799R in the LDLR, which affects the transmembrane domain of the LDLR, prevents membrane insertion and causes secretion of the mutant LDLR. Hum Mol Genet24, 5836-5844, doi:10.1093/hmg/ddv304 (2015).

37 Strom, T. B., Tveten, K., Laerdahl, J. K. \& Leren, T. P. Mutation G805R in the transmembrane domain of the LDL receptor gene causes familial hypercholesterolemia by inducing ectodomain cleavage of the LDL receptor in the endoplasmic reticulum. FEBS Open Bio4, 321-327, doi:10.1016/j.fob.2014.03.007 (2014).

38 Etxebarria, A. et al. Advantages and versatility of fluorescence-based methodology to characterize the functionality of LDLR and class mutation assignment. PLoS One9, e112677, doi:10.1371/journal.pone.0112677 (2014).

39 Soutar, A. K. \& Naoumova, R. P. Mechanisms of disease: genetic causes of familial hypercholesterolemia. Nat Clin Pract Cardiovasc Med4, 214-225, doi:10.1038/ncpcardio0836 (2007).

40 Besseling, J., Kastelein, J. J., Defesche, J. C., Hutten, B. A. \& Hovingh, G. K. Association between familial hypercholesterolemia and prevalence of type 2 diabetes mellitus. JAMA313, 1029-1036, doi:10.1001/jama.2015.1206 (2015).

41 Lazaro, P. et al. Cost-effectiveness of a cascade screening program for the early detection of familial hypercholesterolemia. J Clin Lipido/11, 260-271, doi:10.1016/j.jacl.2017.01.002 (2017).

42 Leren, T. P. Cascade genetic screening for familial hypercholesterolemia. Clin Genet66, 483-487, doi:10.1111/j.1399-0004.2004.00320.x (2004). 
43 Goldberg, A. C. et al. Familial hypercholesterolemia: screening, diagnosis and management of pediatric and adult patients: clinical guidance from the National Lipid Association Expert Panel on Familial Hypercholesterolemia. J Clin Lipido/5, S1-8, doi:10.1016/j.jacl.2011.04.003 (2011).

44 Huijgen, R., Hutten, B. A., Kindt, I., Vissers, M. N. \& Kastelein, J. J. Discriminative ability of LDLcholesterol to identify patients with familial hypercholesterolemia: a cross-sectional study in 26,406 individuals tested for genetic FH. Circ Cardiovasc Genet5, 354-359, doi:10.1161/CIRCGENETICS.111.962456 (2012).

\section{Tables}

Table 1 The results of the blood lipid test of family members

\begin{tabular}{|c|c|c|c|c|c|c|c|c|}
\hline $\begin{array}{l}\text { Family } \\
\text { number }\end{array}$ & ago & Variants & $\begin{array}{l}\mathrm{TC} \\
(\mathrm{mmol} / \mathrm{l})\end{array}$ & $\begin{array}{l}\text { TG } \\
(\mathrm{mmol} / \mathrm{l})\end{array}$ & $\begin{array}{l}\text { HDL-C } \\
(\mathrm{mmol} / \mathrm{l})\end{array}$ & $\begin{array}{l}\text { LDL-C } \\
(\mathrm{mmol} / \mathrm{I})\end{array}$ & $\begin{array}{l}\text { ApoA } \\
(\mathrm{g} / \mathrm{l})\end{array}$ & $\begin{array}{l}\text { ApoB } \\
(\mathrm{g} / \mathrm{l})\end{array}$ \\
\hline I1 & 77 & p.Gly343Ser & 8.75 & 0.67 & 1.28 & 7.27 & 1.34 & 0.45 \\
\hline 12 & 75 & p.Ala627Thr & 6.81 & 2.12 & 0.93 & 5.31 & 1.16 & 1.32 \\
\hline II2 & 53 & $\begin{array}{l}\text { p.Gly343Ser, } \\
\text { p.Ala627Thr }\end{array}$ & 20.50 & 1.45 & 1.26 & 18.80 & 1.53 & 0.97 \\
\hline II3 & 56 & - & 2.94 & 0.76 & 1.35 & 1.42 & 1.51 & 0.40 \\
\hline II5 & 50 & - & 3.61 & 0.46 & 2.11 & 1.37 & 1.76 & 0.44 \\
\hline 117 & 48 & $\begin{array}{l}\text { p.Gly343Ser, } \\
\text { p.Ala627Thr }\end{array}$ & 21.35 & 2.27 & 0.58 & 20.29 & 1.20 & 1.45 \\
\hline III1 & 26 & p.Gly343Ser & 6.62 & 1.22 & 1.12 & 4.92 & 1.10 & 1.21 \\
\hline III2 & 28 & p.Gly343Ser & 8.81 & 1.02 & 1.10 & 7.48 & 1.34 & 1.17 \\
\hline III3 & 30 & - & 3.60 & 1.53 & 0.63 & 2.49 & 1.16 & 0.93 \\
\hline III4 & 30 & - & 3.27 & 0.93 & 1.10 & 2.04 & 1.31 & 0.62 \\
\hline III5 & 23 & - & 3.18 & 1.16 & 1.03 & 1.94 & 1.19 & 0.73 \\
\hline III6 & 27 & - & 3.06 & 1.38 & 0.76 & 2.02 & 0.93 & 0.70 \\
\hline III7 & 22 & p.Ala627Thr & 7.58 & 1.38 & 1.08 & 6.11 & 1.24 & 0.82 \\
\hline III8 & 20 & p.Gly343Ser & 6.97 & 0.87 & 1.68 & 5.03 & 1.61 & 0.54 \\
\hline $\begin{array}{l}\text { Normal } \\
\text { reference } \\
\text { value }\end{array}$ & & & $\varangle 5.18$ & $\otimes 1.7$ & $\begin{array}{l}1.04- \\
1.55\end{array}$ & $0-3.37$ & $\begin{array}{l}1.2- \\
1.6\end{array}$ & $\begin{array}{l}0.6- \\
1.1\end{array}$ \\
\hline
\end{tabular}

Note: TC, Total cholesterol; TG, Triglycerides; HDL-C, High density liptein cholesterol; LDL-C, Low Density Lipoprotein; apoA, Apolipoprotein B; apoB, Apolipoprotein B 
Figures
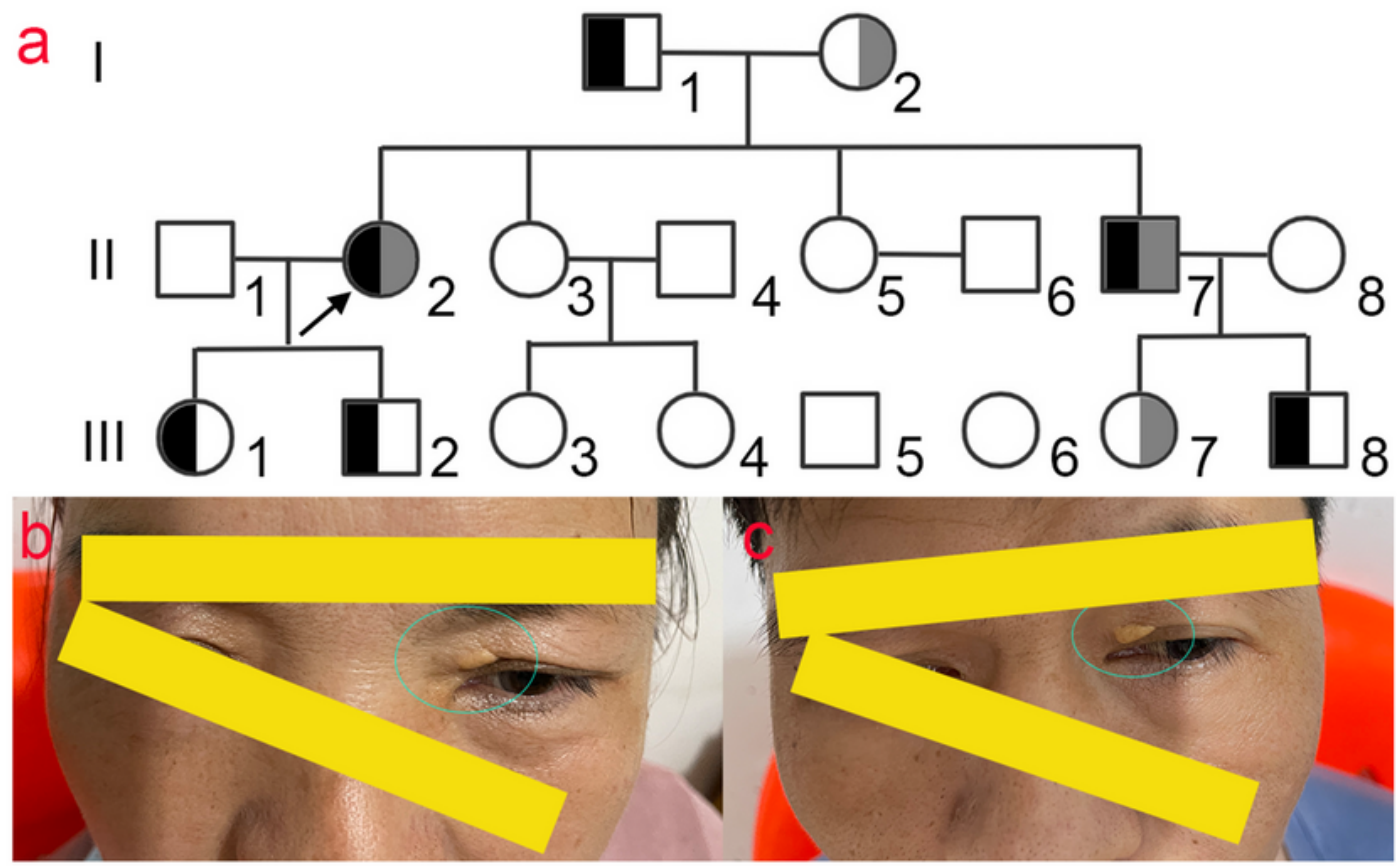

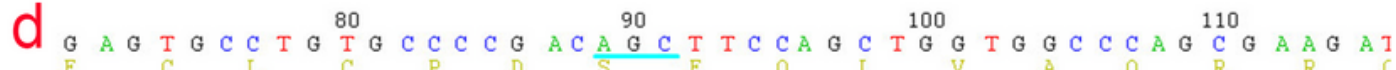
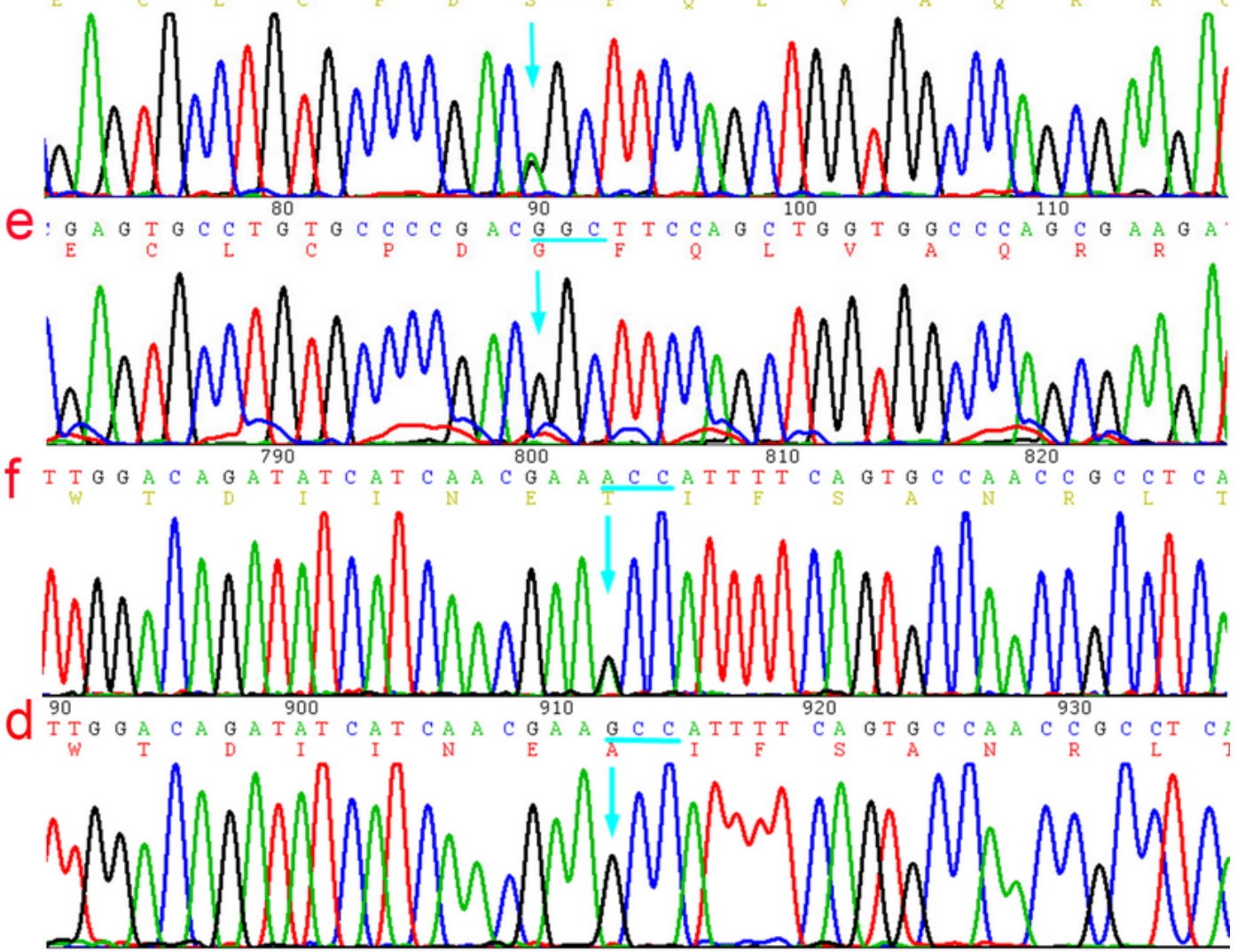

Figure 1

a) Family genogram of $\mathrm{FH}$. The proband (II2) and younger brother (II7) carrying compound heterozygotes; black and grey indicate c.G1027A (p.G343S) and c.G1879A (p.A627T) mutation in LDLR gene, respectively. b) and c) The symptoms of xanthomas on the eyelid skin of the proband (left) and brother 
(right). d) The Sanger sequencing map of the c.G1027A (p.G343S) mutant and e) The corresponding wild-type Sanger sequencing map. f) The Sanger sequencing map of the c.G1879A (p.A627T) mutant and g) The corresponding wild-type Sanger sequencing map.

\section{Figure 2}

Effects of LDLR Gly343Ser and Ala627Thr mutations on the protein tertiary structure. a) The LDLR interacting proteome included in the String database (https://string-db.org/cgi/network? taskld=bniuzKD4qISX\&sessionld=b8YfT7vyWuWv). b) The tertiary structure of LDLR (green) and its interacting protein PCSK9 (yellow and purple) was obtained from Swiss-model database (https://swissmodel.expasy.org/repository/uniprot/P01130). c) Chimera 1.15 (https://community.chocolatey.org/packages/chimera/1.15) predicted changes in LDLR tertiary structure by the mutation of Gly343 to Ser343. d) Chimera 1.15 predicted changes in LDLR tertiary structure by the mutation of Ala627 to Thr627. 


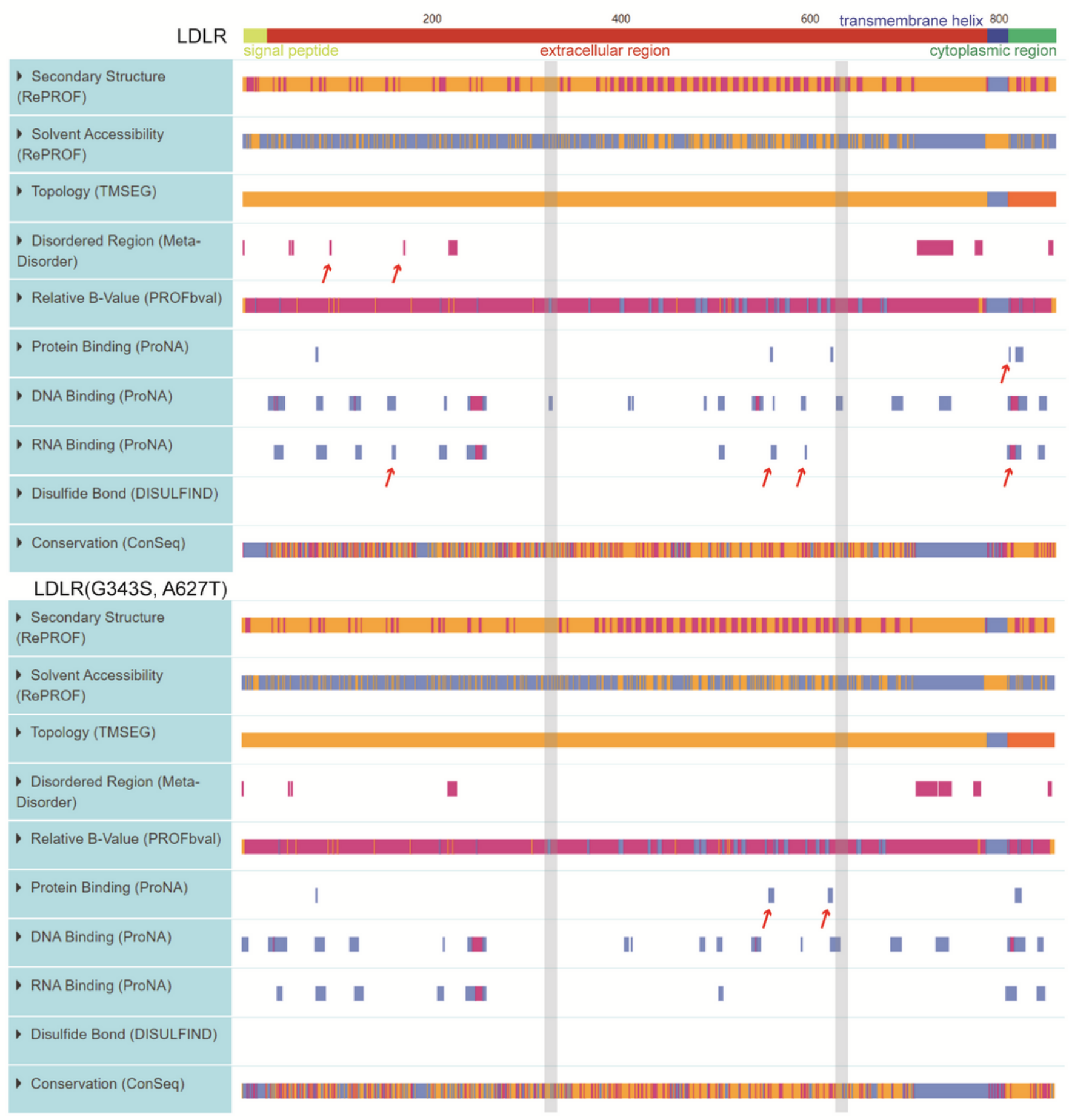

\section{Figure 3}

Effects of LDLR Gly343Ser and Ala627Thr mutations on protein functional domains. Functional domain prediction for wild-type (top) and mutant (bottom) using Predictprotein (https://predictprotein.org/). The shaded area represents the location of the mutation; the red arrows indicate regions where wild-type and mutants vary significantly in functional domains.

\section{Figure 4}


Effects of LDLR Gly343Ser and Ala627Thr mutations on protein post-translational modification. a) Conservation analysis of sequences near the mutation sites of Gly343Ser and Ala627Thr. * indicates pathogenic mutation loci included in the Uniprot database (https://www.uniprot.org/uniprot/P01130). b) GPS 5.0 (http://gps.biocuckoo.cn/) predicted signaling pathway regulating phosphorylation of Gly343Ser mutation site. c) GPS 5.0 predicted the phosphorylation signaling pathway function on the Ala627Thr mutation site. d) GPS 5.0 predicted the effect of Ala627Thr mutation onphosphorylation signaling pathway of the Ser630 site. e) YinOYang-1.2 (https://services.healthtech.dtu.dk/service.php?YinOYang1.2) predicted the effect of LDLR Gly343Ser and Ala627Thr mutations on protein O-glycosylation

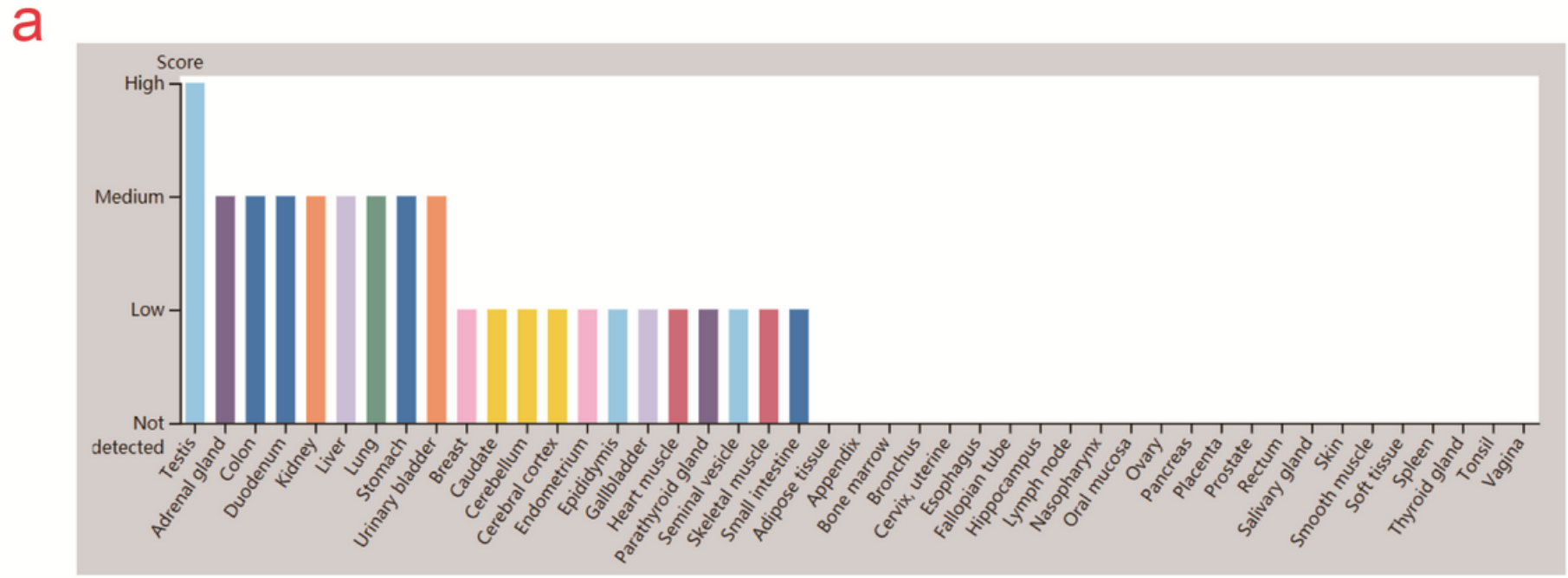

b

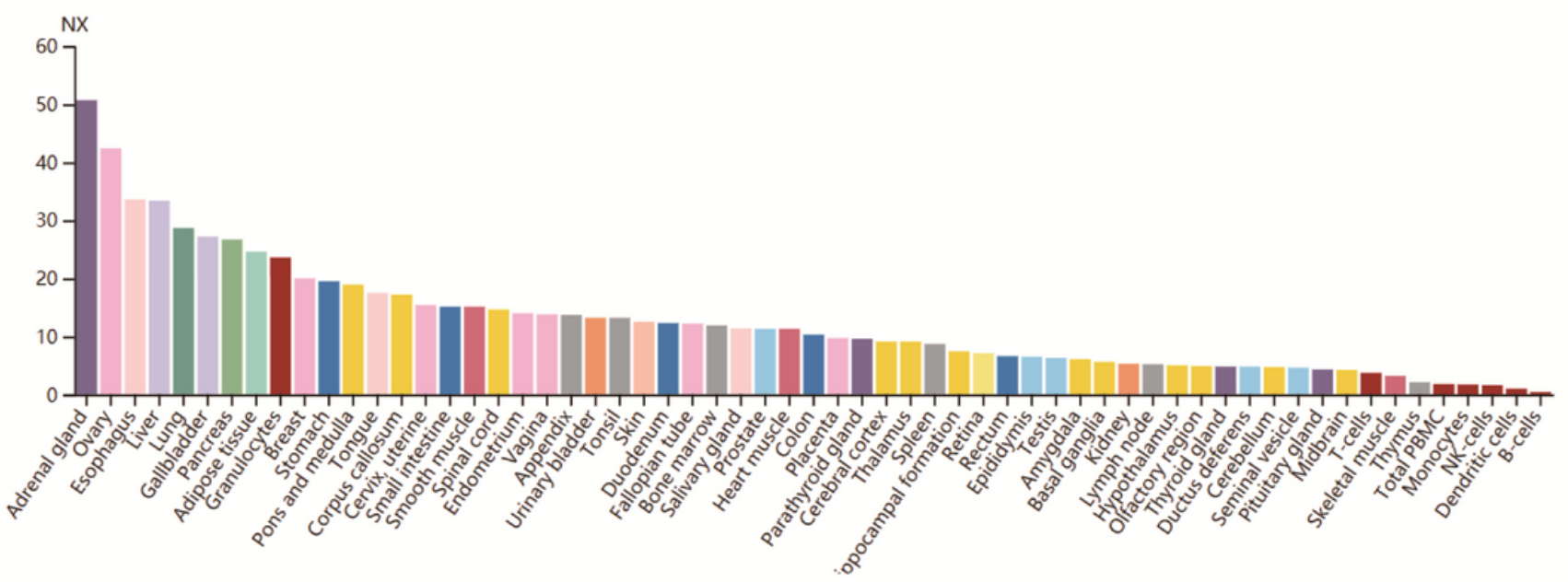

C

\begin{tabular}{|l|l|l|}
\hline Knockout phenotype & Rank & Author(Year) \\
\hline induce spontaneous cholesterol depletion & $5 / 20,564$ & van den Boomen DJH (2020) \\
\hline decreased viability in cells latently infected with KSHV & $27 / 11,468$ & Holmes DL (2020) \\
\hline prevents transport of LDL-derived cholesterol to the ER & $38 / 19,090$ & Trinh MN (2020) \\
\hline increased resistance to SARS-CoV-2 & $52 / 20,914$ & Wang R (2020) \\
\hline
\end{tabular}

\section{Figure 5}


Expression and functional analysis of LDLR. a-b) The Human Protein Atlas database (https://www.proteinatlas.org/ENSG00000130164-LDLR) includes the expression levels of LDLR mRNA (a) and protein (b) in various tissues and cells. c) The CRISPR high-throughput screening database BioGRID (https://orcs.thebiogrid.org/Gene/3949) includes the physiological and pathological functions that LDLR is mainly involved in.

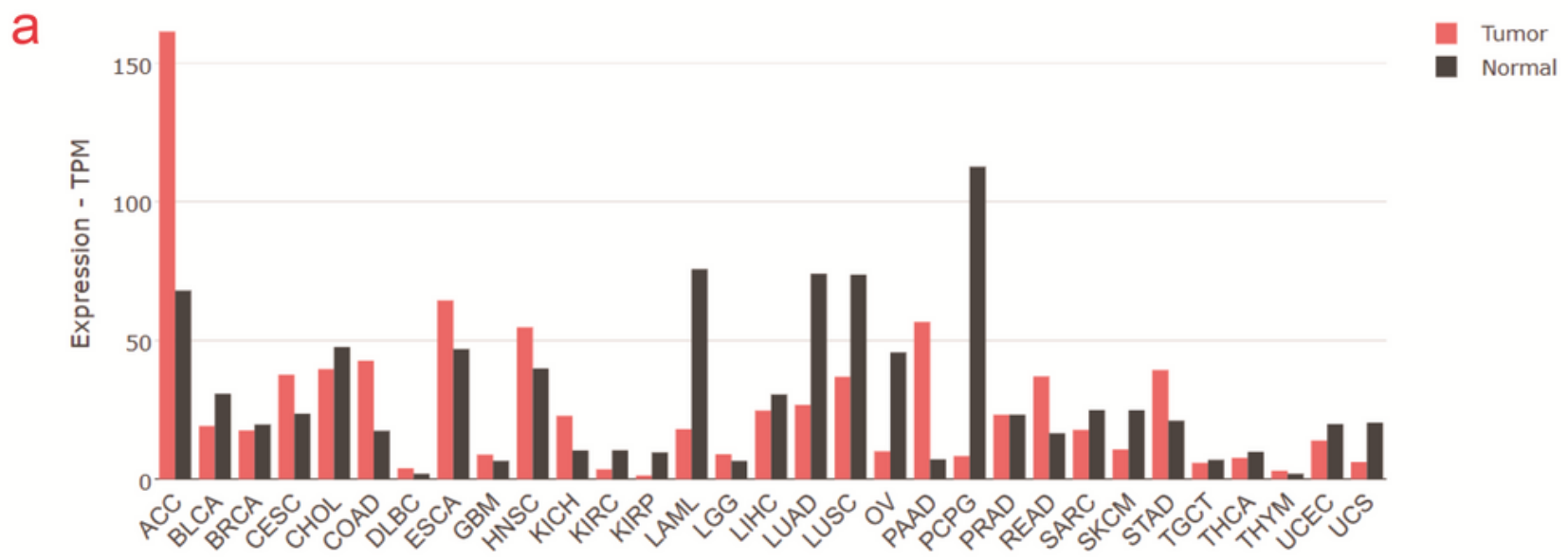

b

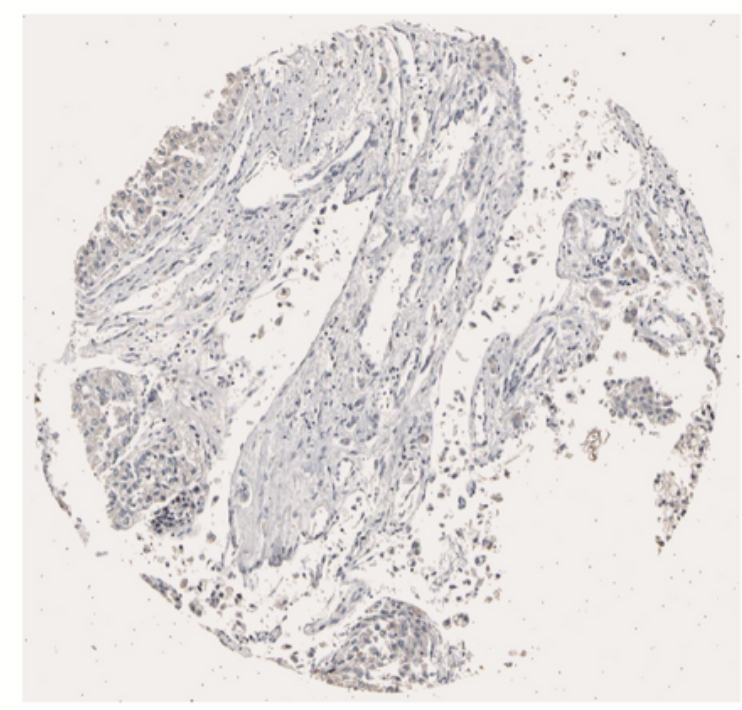

C

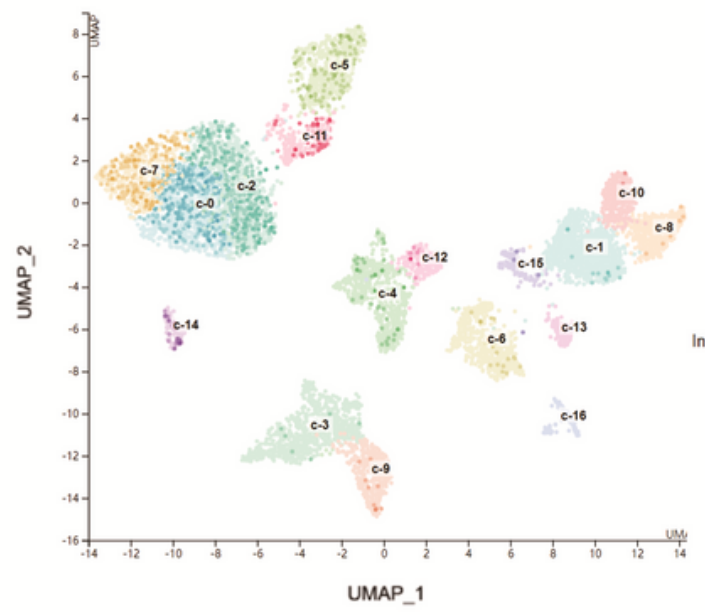

d

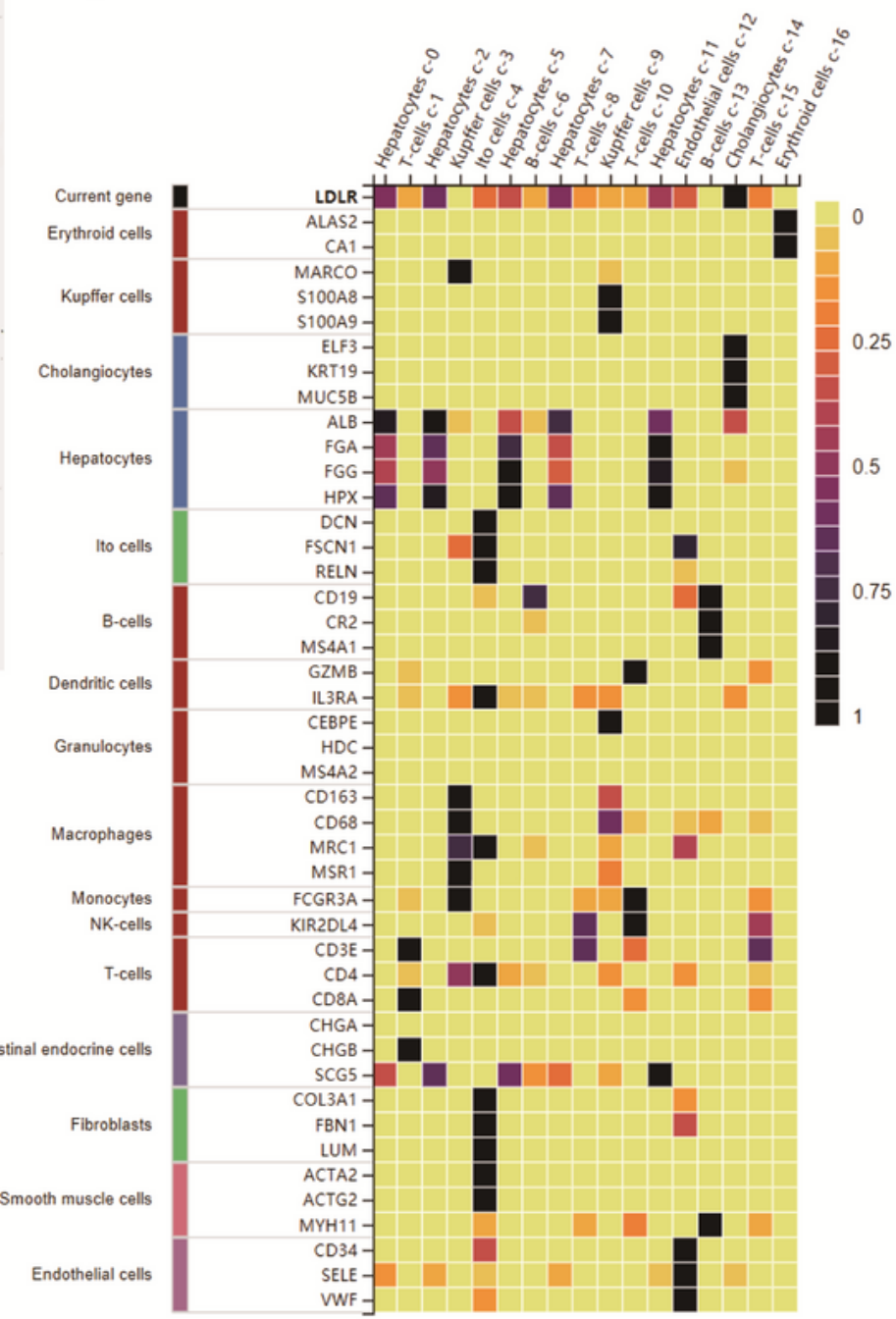

Figure 6 
Relationship between LDLR dysfunction and cancer. a) The GEPIA 2 database (http://gepia2.cancerpku.cn/\#general) includes the expression changes of LDLR in various cancer and normal tissues. b) The Human Protein Atlas database (https://www.proteinatlas.org/ENSG00000130164-LDLR) includes the expression staining of LDLR in hepatoma sections. c) Cell typing of hepatoma using single-cell transcriptome sequencing data. d) Single-cell transcriptome sequencing to verify the expression of LDLR in various hepatoma cells.

\section{Supplementary Files}

This is a list of supplementary files associated with this preprint. Click to download.

- Supplement1.tif 